\title{
El equilibrio ecológico mundial y los países subdesarrollados
}

Cientista político brasileño, es autor de diversos libros, entre los cuales destacan Economic and Political Development, Harvard University Press, 1968, y La Dependencia Politicoeconómica de América Latina, Siglo xx, 1970, en colaboración con otros autores. $\mathrm{Su}$ último libro, Political Development, está siendo impreso actualmente por Harper \& Row. Jaguaribe fue profesor de las Universidades de Harvard, Stanford y el MIT, y actualmente pertenece al Instituto de Pesquisas de Río de Janeiro.

\section{NOTA DE LA REDACGIÓN}

Este articulo, que se publica en su versión preliminar, contiene una breve discusión de las tesis del Club de Roma, particularmente en lo que se refiere a sus aspectos politicos y a su significado para el Tercer Mundo. La versión definitiva de este ensayo deberá aguardar la conclusión de los trabajos del Club de Roma. El Club de Roma se gestó en abril de 1968, cuando 30 personalidades-cientificos, economistas, educadores, humanistas y hombres públicos- se reunieron en la Accademia dei Lincei, en Roma, a instigación del economista $y$ empresario italiano, doctor Aurelio Peccei. La asociación internacional de intelectuales y cientificos que surgió de esa reunión se consagró al estudio de la problemática mundial-crecimiento exponencial de la población, la industrialización y el consumo; degradación del medio ambiente; pobreza en medio de la abundancia; bancarrota de las estructuras urbanas; falta de fe en las instituciones; alienación de la juventud, y otras graves disrupciones- en la convicción de que cada una de las variables mencionadas contiene aspectos políticos, técnicos, económicos, sociales y culturales que interactuan entre si, por lo que sólo pueden ser abordadas en forma interdisciplinaria. El Club de Roma, una organización informal que ha sido adecuadamente descrita como una "universidad invisible", cuenta hoy con setenta miembros de veinticinco nacionalidades y ha dado a la publicidad sus primeras conclusiones, principalmente a través de los libros The Limits to Growth y Blueprint for Survival. El profesor Jaguaribe reficre su análisis a los modelos predictivos contenidos en dichas publicaciones. 


\section{LA PROBLEMIATICA MUNDIAL}

\section{PROPÓSITO DEL PRESENTE TRABAJO}

El objetivo del presente trabajo es el de llevar a cabo una breve discusión acerca de la tesis principal que ha sido desarrollada por el Club de Roma (CoR), incluyendo sus intentos de construir un modelo global que exprese las principales variantes que interfieren en la relación entre el hombre y su medio ambiente natural. Tales variantes y sus interrelaciones han sido consideradas por el CoR en una amplia perspectiva de manera que, dados los datos y ciertas suposiciones, su comportamiento en el pasado, desde el comienzo del siglo, y su comportamiento en el futuro, a través del final del próximo siglo, pueden ser simulados en computadoras.

Es el propósito de este estudio el presentar inicialmente, en esta sucinta Primera Parte los puntos de vista centrales del Club de Roma. Posteriormente se hará un intento de indicar en la Parte II las áreas principales de problemas que comprenden tales tesis y comentar rápidamente algunos de esos problemas. Finalmente, se llevará a cabo una discusión en la Parte III, también en forma condensada, de los aspectos políticos de esa problemática, particularmente en vista del presente sistema internacional y de la situación de los países subdesarrollados.

\section{LA TESIS GENTRAL DEL COR}

La tesis central del Club de Roma ${ }^{1}$ es que los efectos combinados del actual crecimiento exponencial de la población mundial y de su consumo per cápita están acumulando una presión sobre los recursos limitados de la tierra y sobre la limitada capacidad de autoregulación y auto-regeneración del ecosistema natural del hombre, la que necesariamente traerá como consecuencia, en un futuro no remoto (hasta 60 años), un reequilibrio general de la relación entre el hombre y su ecosistema natural a expensas de un decrecimiento catastrófico de la población mundial, debido a la mortandad masiva, y de una profunda degradación del estándar material y cultural de la humanidad, excepto si se adoptan medidas apropiadas a tiempo.

Lo que el CoR prevé es la inevitabilidad, en algún momento entre la tercera y cuarta década clel próximo siglo, de un equivalente

${ }^{1}$ Cf. Ia serie de artículos de Aurelio Peccei en Successo, febrero y junio de 1970 y particularmente febrero de 1971, pp. 129-139. 
tecnológico, para todo el mundo y en una escala incomparablemente más trágica, de la Peste Negra del siglo XIV en Occidente, si no se introducen a tiempo cambios drásticos para proteger la biósfera y para prevenir el aniquilamiento de recursos naturales no renovables.

Para alcanzar esta conclusión el CoR comienza enfatizando la necesidad de comprender los muchos problemas que resultan del crecimiento exponencial de la humanidad, tanto en números como en capacidades económico-tecnológicas y en los crecientes anhelos en términos de una enorme, compleja e intrincada problemática del mundo ${ }^{2}$. Tal problemática no puede ser ya alcanzada en términos de disciplinas académicas aisladas o tratada por partes, o en función de desconectados intereses particulares, nacionales o económicos. Su enfoque y tratamiento debe necesariamente ser 1) sistemático, 2) global, 3) diacrónico y 4) normativo, en el sentido de conducir al establecimiento de regulaciones factibles de ser impuestas. El enfrentamiento del hombre con tal problemática le presenta la ineludible alternativa de entenderla y someterla a un control racional y planeado o ser la víctima de un reajuste natural catastrófico. Tal es el dilema de la humanidad ${ }^{3}$.

\section{LOS MODELOS GLOBALES}

Para poder clominar la intrincada complejidad de las muchas variables que intervienen, interrelacionadas entre sí, el CoR adoptó la técnica de la "Dinámica de Sistemas" inicialmente desarrollada por el profesor Jay Forrester en el M.I.T., en la cécada del sesenta, para el estudio de complejos sistemas industriales. La técnica de la dinámica de sistemas permite la construcción de complejos modelos dinámicos representacionales que pueden ser tratados por computadoras digitales, cladas ciertas suposiciones, que expresan el comportamiento de las variables interrelacionadas en un período de tiempo dado.

Después de un intento preliminar de construir y expresar tales variables (World 1) dos modelos globales (World 2 y World 3), con 40 y 60 variables interrelacionadas respectivamente, han sido diseñados y experimentados. Las variables principales consideradas por los modelos fueron la población mundial y su crecimiento tendencial, tal como lo expresan los indices de nacimiento y mortalidad; la obtenibilidad de recursos naturales no renovables, alimentos percápita expresados por la obtenibilidad de tierra potencialmente la-

"Cf. Aurelio Peccei "The Predicament of Mankind" en Successo, junio de 1970, pp. 140-150.

${ }^{3}$ Aurelio Peccei, op. cit., p. 154. 
brable, capital agrícola, alimentos extraíbles de los mares y otros factores; la contaminación y su ritmo de crecimiento; el estándar nacional de vida y la calidad de la vida, en función de la relación de estrechamiento y otras variables 4 .

La población mundial, que se estimaba en tres billones para 1960 y 3.5 billones en 1970 se está duplicando cada 32 años, con una tendencia a crecer en forma aún más rápida. Esto significa, frente a la proyección media anterior de la UN de 6.4 billones para el año 2000, que en el cambio de siglo la población mundial será de alrededor de 7 billones.

No es solamente la población la que cuenta sin embargo, sino. el producto del número de seres humanos por sus capacidades económico-tecnológicas de extracción y consumo de items renovables y no renovables, en función de sus aspiraciones y estilos de vida, incluyendo los usos que conciernen a la mayor o menor duración de bienes durables, el destino dado a los desperdicios sólidos, el tratamiento dado a las emanaciones fluidas industriales, etc. Aurelio Peccei llama a ese producto del número de gente por su efectiva capacidad de consumo (estimada en función del PBN per cápita) la presión demográfica réal. Si se consideran los indices de la presión demográfica real, más bien que los simples números de población, tenemos. que mientras la presión demográfica real en 1900, fue de 4:12 (población -,5 billones), aumentó en 1966 (población 3.4 billones) a. 3.483 y alcanzará, en el 2000 (considerando la estimación de la UN de 6.4 billones de personas) a 28.700 , lo cual representa, para un siglo, frente a la multiplicación de la población por alrededor de 4, una multiplicación de la presión demográfica real por más de $70^{5}$.

Suponiendo las proporciones actuales de consumición de los recursos naturales no renovables de crecimiento demográfico, relación. alimenticia, relación de contaminación y las actuales direcciones. concernientes a otras variables claves, que corresponden a su desarrollo estándar, el modelo World 2 del CoR, simulando el comportamiento de aquellas variables desde 1900 , señala que un pico de población se alcanzaría alrededor del año 2030 y comenzaría luego. a declinar, suavemente primero, y catastróficamente a partir de alrededor del año 2040 en adelante, debido al efecto depresor de la declinación de los recursos naturales, seguido por un terrible aumento. de contaminación ${ }^{6}$.

El mismo modelo World 2 ha sido experimentado por el CoR. en función de algunas suposiciones alternativas relevantes. Por ejem-

"Cf. Dennis L. Meadows, informe (mimeografiado) a la reunión del G. deR. en Montebello, abril de 1971, "The Dynamics of World Equilibrium", p. 11, sig. ${ }^{5} \mathrm{Cf}$. Aurelio Peccei, op. cit., pp. 132-153.

${ }^{\circ}$ Cf. Dennis Meadows, op. cit., pp. 20-2I. 
plo, el desarrollo 12S, en el cual se suponía que adelantos decisivos (tomando en cuenta la continuidad de los ciclos de desperdicio y de nuevos avances tecnológicos) reduciría el uso de recursos naturales a un $25 \%$ de su valor previo en el año 1970, si los demás factores se mantienen. En tal caso la decadencia de recursos naturales ya no oprimiría a la población. "En cambio, la población y el capital continúan aumentando hasta que una crisis del medio ambiente surja cle la contaminación y se afirme a sí misma". El pico de población se alcanzará por el año 2040 y una aguda reducción de población debido a una muerte masiva por contaminación, llevará el nivel demográfico, hacia el 2100, por debajo del nivel de $1900^{7}$.

En otro grupo de hipótesis, World 2-22S, se considera, como en el anterior, que la utilización de recursos naturales se reduce a un $25 \%$ de su proporción previa. Más aún, la generación de inversión de capital se aumenta un $20 \%$ y la generación de contaminación se reduce de 1 a 0.5 . El resultado final es simplemente una postergación, por alrededor de 10 años, de la crisis indicada en el desarrollo anterior. La reducción de la utilización de recursos naturales y de la producción de contaminación permite a la población y a la inversión de capital aumentar lo suficiente para finalmente anular las ventajas de tales reducciones, restableciendo una situación de crisis y su reequilibrio por el incremento masivo de la proporción de muerte y el correspondiente decrecimiento de población, con una caída igualmente dramática de la calidad de la vida.

La simulación producida por el modelo más sofisticado World 3, más parecido que el anterior a la vida real, no es diferente en clase. El punto más alto de población se alcanza alrededor de 2070 (más - menos trece billones), con una declinación abrupta desde cerca del 2080 en adelante. Los índices de mortalidad, debiclos a la escasez de alimentos y a la escasez de los recursos naturales, comienza a aumentarse desde alrededor del 2025, acelerándose rápidamente hacia el 2050 en adelante.

\section{LAS PRINGIPALES CONCLUSIONES PRELIMIINARES DEL COR}

De acuerdo al profesor Dennis Meadows ${ }^{2}$, los experimentos con el modelo World 3 han conducido, con muy poco desacuerdo, a las siguientes conclusiones preliminares:

־Cf. Dennis Meadows, op. cit., pp. 22-23.

${ }^{8} \mathrm{CF}$. Dennis Meadows, op. cit, pp. 24-25.

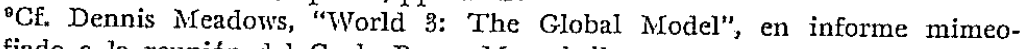
grafiado a la reunión del $\mathrm{C}$. de $\mathrm{R}$. en Montebello. 
1. No hay posibilidad de suficiente progreso tecnológico y cultural en los próximos 100 años para manterier un número de 14 billones de personas en nuestro globo. Ya que el tiempo de duplicación de población es corrientemente 32 años en forma decreciente, ello significa que en algún momento dentro de los próximos 60 años el crecimiento de la población "sufrirá una desaceleración profunda.

2. No hay posibilidad de que la vasta mayoría de aquellos que viven en los países en desarrollo alcancen el nivel material de vida de que gozan las naciones desarrolladas.

3. Hay una posibilidad fuerte de que las riaciones occidentales observarán una declinación marcada en su propio nivel material de vida dentro de las próximas 3 ó 4 décadas.

4. No hay un nivel único óptimo de población a largo plazo. Más bien hay un conjunto de intercambios entre la libertad personal, el nivel de vida material y social, y el nivel de población. Dado el conjunto finito de recursos, y en disminución, del mundo, nos enfrentamos inevitablemente con la necesidad de reconocer que más gente implica un nivel de vida más bajo.

5. En teoría no hay un valor humano fundamental que no pudiera ser mejor logrado a través de una baja sustancial de la población mundial.

6. Hay una probabilidad muy fuerte de que la transición hacia el equilibrio del globo comprenderá una declinación traumática en la población. Es nuestra conclusión que el deber del "Club de Roma" es identificar y complementar el conjunto de políticas que nos permitirán negociar una transición ordenada hacia el equilibrio. Debemos producir una transición que, aunque produzca cambios drásticos, nos dejará con la mayor parte de los atributos que valoramos en nuestra sociedad y que preservará opciones para aquellos que deben vivir en este globo de aquí a más de un siglo.

\section{LAS PRINGIPALES GUESTIONES ENGARADAS}

\section{CUATRO ÁREAS PRINGIPALES}

Los estudios y conclusiones del CoR comprenden cuatro áreas principales:

1) La naturaleza lógica y epistemológica de las conclusiones del CoR. 
2) El carácter socio-histórico de nuestro tiempo y de su desarrollo futuro más probable, incluyendo su parámetro físico;

3) El problema del equilibrio ecológico mundial en función del sistema internacional;

4) El problema del equilibrio ecológico mundial en función del desarrollo del Tercer Mundo.

Como se ha dicho inicialmente en este trabajo, su propósito principal es discutir, particularmente en función de la situación del Tercer Mundo, la implicación política de los estudios y conclusiones del CoR. Esta discusión, centrada en las clos últimas áreas arriba indicadas será tratada, en forma breve, en la Parte II de este trabajo. Un breve comentario, sin embargo, debe hacerse sobre las dos primeras áreas. En los tópicos siguientes el carácter lógico-epistemológico de las conclusiones del CoR, incluyendo las cuestiones relacionadas con el significado Neo-Maltusiano de algunas de ellas, será rápidamente considerado. Por esto, un comentario sucinto del carácter socio-histórico de nuestro tiempo será también presentado, incluyendo observaciones sobre su parámetro físico.

\section{EL PROBLEMA LÓGICO-EPISTEMOLÓGICO}

La primera de las áreas ya mencionadas concernientes a la naturaleza lógico-epistemológica de las conclusiones del $\mathrm{CoR}$, se refiere a la significación analítica y empírica de tales conclusiones, ¿Qué clase de conclusiones son aquéllas? ¿Pertenecen al campo de las ciencias naturales y son o deberían ser generalizaciones tipo ley de carácter físico-químico? ¿Pertenecen al campo de las ciencias sociales y representan o deberían representar tendencias socio-económicas? ¿O pertenecen al campo de la historia y expresan procesos estructurados pero abiertos?

Yo sugiero que los problemas relativos a la caracterización lógico-epistemológica de las conclusiones del CoR deben ser, en último análisis, reducidos a dos tipos de investigación de validez. El primero se relaciona a) con la validez de la identificación y enumeración de las variables que intervienen y b) con la validez del modelo diseñado para representar el sistema empírico correspondiente.

Muy sucintamente, los principales problemas implicados, en ese aspecto, son los siguientes:

A) RELATIVO A LAS VARIABLES Y SU REPRESENTAGIÓN EN EL MODELO

I. Identificación de variables: validez de la identificación conceptual de cada variable, incluyenclo su caracterización como un fenómeno natural, sociológico o histórico; 
ITelio Jaguaribe / Sil equilibrio ecológico mundial y los paises subdesarrollados

II. Descripción de variables: validez de la observación y medición de las propiedades empíricas y/o comportamiento de cada variable;

III. Representación de variables: validez de la forma de representación de cada variable en el Modelo adoptado;

B) RELATIVO AL MODELO ADOPTADO:

I. Adecuación del Modelo descriptivo: validez del sistema ideal adoptado para describir el sistema empírico que corresponda;

II. Adeculación del modelo explicativo: validez de las interrelaciones adoptadas para las variables del Modelo, frente al sistema empírico.

La segunda investigación se refiere a la determinación, en función del carácter de cada variable adoptada por el Modelo, del tipo de generalizaciones válidas que puede y debe darse a las proposiciones concernientes a su comportamiento. Aquí viene la discriminación de aquellas generalizaciones a las que puede y debe darse un carácter de ley, en términos de una proposición de ley natural, de aquellas que deben ser tratadas como tendencias "estocásticas", en términos de las ciencias sociales y de aquellas que deben considerarse como propensiones históricas abiertas.

No es el objeto del presente trabajo, como ya se dijo, seguir una discusión lógico-epistemológica de las conclusiones del CoR. Limitaré mi intervención en esta área a dos comentarios generales y la observación de un carácter específico. El primer comentario intenta recalcar la absoluta necesidad, en el estado presente de los estudios del GoR y como pre-requisito para su desarrollo ulterior y discusión significativa, de proceder a un análisis lógico-epistemológico riguroso del carácter y la validez de sus conclusiones iniciales. Existe hoy en día una peligrosa propensión a suponer que las técnicas de análisis de sistema y el diseño y procesamiento de modelos computadorizados son substitutos de una crítica lógico-epistemológica de las variables de que se trata y que automáticamente aseguran la validez de proposiciones computadorizadas sobre tales variables. Por cierto, esto no es verdad.

Mi segundo comentario se referia, también en una forma muy general y como una aplicación del anterior, al grupo de conclusiones preliminares presentadas en el informe del Prof. Meadows. Estas conclusiones han sido formuladas como generalizaciones tipo ley de ciencias naturales, para los items I y 2; como generalizaciones sociológicas, para los items 4 y 5 ; y como probables tendencias históricas, para los items 3 y 6 . No hay indicación, en todo el estudio, de nin- 
gún intento de afirmar analíticamente el carácter de tal generalización y parecería que tal tipo de cuestiones aún no ha sido contemplado.

- Esto significaría que su carácter natural, sociológico o histórico, como ya se mencionó, no ha sido considerado críticamente. El riesgo de una clasificación errónea en que se incurre por tales formulaciones no críticas no puede ser despreciado. Tal, por ejemplo, si contemplamos el caso del ítem I de las conclusiones y aún más probablemente el caso del ítem 2.

Esto no quiere decir que dejar que la población del globo crezca a 14 billones no pueda ser un hecho lleno de las implicaciones más negativas para la humanidad -como lo indican los estudios del CoR es muy posible que así sea- tendientes a causar resultados catastróficos. Significan simplemente que generalizaciones sobre ese tema -en lo que respecta al estudio del Prof. Meadows- no parecen presentar las características de una ley natural sino que contienen elementos que las introducen en el campo de las generalizaciones socio-históricas. El darles su propio carácter socio-histórico de ningún modo afecta su importancia, ya sea para propósitos cognoscitivos o normativos. A la inversa, tratar como ley natural una generalización socio-histórica válida la expone al riesgo de que la cliscusión de su pretendida universalidad y necesidad lleve a olvidar su contenido de verdad.

Del mismo modo, pero en un caso aún peor, el ítem 2 formula una generalización similar a una ley, referente a países subdesarrollados que no se adecuan a un tratamiento tal. Los procesos que se refieren al desarrollo de la sociedad ciertamente no son reducibles a fenómenos naturales y si es verdad que asegurar a la vasta mayoría de aquellos que viven en países subdesarrollados el estándar material de que gozan las naciones desarrolladas está lejos de..ser una tarea fácil, uná generalización sobre ese punto no puede ser formulada científicamente- sin introducir varias calificaciones esenciales y un tratamiento probabilístico, ya que incluye tantos factores y condiciones cambiantes y algunos aspectos imprevisibles.

Una última observación sobre este tema, relacionada con la sub. secuénte discusión de los estudios y conclusiones del CoR, concierne a la necesidad, además de la crítica lógico-epistemológica señalada más arriba, de establecer la exactitud factual o la razonabilidad de todos los datos empíricos relevantes y supuestos adoptados por estos estudios. El presente trabajo, que se ocupa principalmente de las im. plicaciones sociopolíticas de estos estudios, no estuvo orientado hacia tales comprobaciones y toma sin discutir el valor de todas las suposiciones fácticas adoptadas por el CoR excepto cuando se afirma lo contrario. 


\section{MALTUSIANISMO ECOLÓGICO}

Algunas de las tesis del CoR, como ya se observó, tienen un significado neo-Maltusiano (como los items 1 y 2 de las conclusiones de Meadows) y el argumento central del CoR (la tasa corriente de incremento de la presión demográfica real tiende a sobrepasar la capacidad autorregulaclora y autorregenerativa del ecosistema natural del hombre) es el equivalente a una formulación del principio Maltusiano en términos ecológicos más amplios.

EI principio de Malthus, como es bien conocido, es uno de los temas más debatidos en los campos de la demografía y la economía. Ha suscitado y sigue suscitando las controversias más apasionadas, y ha tendido, descle la formulación de Nalthus, a atraer el apoyo de conservadores y la oposición de progresistas. No es objeto del presente trabajo concentrarse en la discusión de ese problema. Solamente presentaré algunos pocos comentarios sobre él, en conclusión a las observaciones lógico-epistemológicas del tópico precedente.

Malthus, como ya se sabe desde hace tiempo, no inventó "su" principio, que había sido originaria y completamente formulado por Giovanni Botero ${ }^{10}$ y discutido por muchos otros autores antes que Malthus. El éxito de Malthus se debió a la oportunidad histórica de su formulación (ignorando a sus predecesores) de tal principio, el comienzo de la revolución industrial. La construcción como pseudoley que Malthus inicialmente dio a ese principio ha contribuido poderosamente, primero, a șu populariclad y, con el correr del tiempo, a su controversialidad. Botero había sostenido que la población tiende a aumentar hasta el límite de la fecundidad humana (virtus generativa) mientras que las posibilidades de aumentar los medios de subsistencia (virtus nutritiva) son limitadas y por eso imponen un límite a tal aumento, el único. que hay. Este límite se hace sentir a través de necesiclades (escasez) que inducirán a la gente a evitar el matrimonio (control negativo de Malthus, limite prudencial y "continencia moral") a menos que la cantidad sea periódicamente reducida por guerras, peste, etc. (el control positivo de Malthus) ${ }^{11}$. La primera enunciación del principio de Malthus en la primera edición anónima (1798) de su Ensayo sobre el Principio de la Población fue hecha en forma de una ley: la población crece en proporción geométrica mientras que los medios de subsistencia crecen en proporción aritmética. La población, por eso, tiende a

${ }^{10}$ En el libro de Botero Delle Cause della Grandezza della Gittd, 1588.

${ }^{12}$ Ver la discusión del tema por Joseph Schumpter, en su History of Economic Analysis, pp. 252, 254-258, 278-284. 
exceder a la subsistencia. Si no se la controla y limita con prudencia, solamente será limitada con controles positivos tales como la guerra, el hambre, las enfermedacles, la miseria y el vicio. En la segunda edición cle su trabajo (1803), revisada y ampliada, Malthus suprimió las tasas que pretendían ser matemáticas y formuló su principio en tres puntos:

a) La población necesariamente está limitada por los medios de subsistencia.

b) La población invariablemente aumenta mientras que los medios de subsistencia aumentan a menos que impedimentos poderosos y obvios los detengan.

c) Todos estos impeclimentos (o controles) pueden resolverse en continencia moral, vicio, miseria.

La primera objeción contra el pretendiclo carácter natural del principio de Malthus fue presentada por Marx, que apasionadamente se opuso a la tesis de Malthus (a quien detestaba profundamente) diciendo que no hay una superpoblación natural. La superpoblación se debe a la mala distribución de los bienes peculiar al capitalismo'12.

Sin desarrollar un tema que es marginal al propósito del presente trabajo voy a hacer solamente dos comentarios sobre él. El primero se refiere al hecho de que el principio de Malthus (aceptando el hecho histórico de que el nombre de Malthus lo clesigna y no el cle Botero) no es, como Marx correctamente observó, una ley natural, sino una generalización socio-histórica. La relación entre población y medio de subsistencia, como tal, es de tipo biológico natural ya sea una polblación de moscas o de hombres. Lo que imparte un carácter socio-histórico al principio de Malthus, en conjunto, es la forma en que las poblaciones humanas, confrontaclas con problemas de subsistencia, tratan de resolverlos; ya sea aumentando más allá de suposiciones previas la disponibilidad de alimentos, o regulando la distribución cle alimentos de acuerdo a criterios dispares (el argumento de Marx con respecto al capitalismo) o, también, adoptando, cualquier sea el sistema distributivo, otras regulaciones para el control de la población.

El segundo comentario que quisiera presentar se refiere a la valiclez del principio de Malthus, consiclerado como una generalización socio-histórica. En ese sentido sugiero que el principio merece más crédito que lo que Marx y los pensadores progresistas han

"C. Marx Principles of a Critique of Polilical Economy, It. (Ficl. Pleiade, IT, p. 225 sig. y. especialmente solme Malthus, pp. I.52S-1.531). Yer también Capital, vol. I, cap. XXV especialmente sección III (E.d. Pleiade, I, p. 1.141 sig.) 
estaclo dispuestos a reconocer. A grosso modo el problema presenta condiciones distintas de acuerdo a la disponibiladad o no de nuevas fronteras $y / 0$ de innovaciones tecnológicas que permitan el aumento de alimentos en mayor proporción que el crecimiento demográfico. Poco después de MIalthus, por ejemplo, la expansión de la civilización occidental en las Américas y la explotación de Africa, combinados con un constante aumento de innovaciones tecnológicas, ofrecieron a Occiclente la posibilidad de coordinar la dismimución de la mortalidad y el crecimiento demográfico con un aumento en la total obtención de alimentos.

Condiciones distintas se presentaron en la Antigüedad y la Edad Media en Occidente, $y$ lo mismo con respecto a Asia y África antes de ser occidentalizadas. Como to ha indicado Max Weber tan claramente en su estudio de las causas de la decadencia del Imperio Romano $^{13}$, tal decadencia se debió en primer lugar a que los recursos estaban exhaustos una vez que la expansión del imperio llegó a sus límites, teniendo en cuenta la tecnología de que disponían y su organización socio-económica.

Lo que es particularmente importante en los estudios del CoR en lo que respecta a las condiciones existentes en las sociedades industriales y post industriales de hoy, es el hecho de que parece que se han alcanzado nuevos limites de fronteras y tecnologia. Ya no hay más lugares desconocidos en el planeta si bien grancles áreas arables están todavía sin cultivar o explotadas pobremente. No hay posibiliclades razonablemente previsibles de una explotación en gran escala de otros planetas. $Y$, lo que es más importante, hay síntomas alarmantes de que las posibilidades de continuas innovaciones tecjológicas teoréticamente aún abiertas serán contenidas por los limites de la capacidad autoregenerativa y auto-reguladora del ecosistema natural del hombre, agravados por la disminución industrial de recursos no renovables. Para colmo también hay síntomas visibles, que serán considerados en el próximo tópico, de que el hacinamiento de gente y la masificación del mundo (aun cuando regulaciones apropiadas e innovaciones permitiesen un aumento substancial, bastante más allá de los límites previstos por el estudio del Prof. Mea(lows), se volverían otro factor limitacionista de la expansión de la raza humana, o implicarían un alto costo para la mayoría cle los valores y estilos de vida que han sido esenciales a la visión humanista de la buena vida, desde los clásicos griegos, hebreos, chinos o hindúes.

${ }^{13}$ Max Weber, "La decidencia de la Cultura Antigua", traducción española en Revista de Occidente, vol. XIII, 139 37, julio de 1926. (Ha aparecido una traducción inglesa posteriormente en The Journal of Gencral Education, vol. V (195), pp. 75-88). 


\section{EL GARÁCTER SOCIO-HISTÓRICO DE NUESTRO TIEMPO}

El análisis del carácter socio-histórico de nuestro tiempo importaría una discusión más compleja y extensa que el análisis de las implicaciones lógico-epistemológicas de las conclusiones del CoR. En realidad ya existe una amplia literatura sobre el tema que aquí no-podemos ni siquiera mencionar. Dado el propósito de este trabajo el presente tópico sólo contendrá algunas breves observaciones sobre tema tan complejo, solamente para señalar algunos de sus problemas más cruciales, como un esquema de referencias que vuelva más claro el análisis político que se intentará en la Parte. III de este estudio. Con tal objeto voy a dar una breve indicación sobre los conceptos respecto al carácter socio-histórico de nuestro tiempo presentados por algunos de los analistas más agudos. Complementando tales conceptos, el siguiente tópico presentará una referencia aún más sucinta de ideas sobre la posibilidad de obtener recursos materiales, con el fin de extraer algunas conclusiones generales respecto a las condiciones del mundo contemporáneo.

Una de las características más constantes de cualquier análisis competente de nuestro tiempo -entendiendo por tal, en general, el período subsecuente a la primera guerra mundial $y$, particularmente la secuela de la segunda guerra mundial- es el abandono de la confianza optimista y alegre, que prevalecía desde el siglo 18, en el progreso en forma casi lineal de los asuntos humanos. El genio de Oswald Spengler, cualesquiera hayan sido sus errores y abusos especulativos, marcó dicha transición con un libro que expresaba, mucho más que sufrimiento de la Alemania posterior a la guerra, el primer vistazo contemporáneo hacia algunas de las tremendas dimensiones del nuevo mundo del hombre.

Algunas de las características básicas de nuestro tiempo han sido detectadas por Karl Jaspers desde $1930^{14}$, tales como la relación circular entre industrialización masiva y masificación de la sociedad. En sus obras posteriores Jaspers desarrolló y elaboró las implicaciones de sus puntos de vista iniciales. Así dará énfasis, en Origin and Target of History ${ }^{15}$, al hecho de que la dominación de las masas se ha vuelto, al mismo tiempo, un proceso empírico y la nueva condición del hombre. Las tres consecuencias principales que resultan de tal situación son: 1) las masas humanas presionan para conseguir

${ }^{14} \mathrm{Cf}$. The Spiritual Situation of our Time, original alemán edit. 1930, traducción francesa publ. por E. Nauwelaerts, Lluvain y Desclée de Brouwer, París,
1952 .

15Primera edición alemana por R. Piper \& Co. Verlag, Mfunchen, 1949. Trad. española por Revista de Occidente, Madrid, 1950. 
organización y regulación: socialismo; 2) la unidad mundial exige un sistema mundial: Imperio Mundial u orden mundial; 3) la pérdida de creencias tradicionales ya sea hacia el nihilismo o hacia el amor. De modo que lo que está en juego es el destino mismo de la libertad humana, tanto política como espiritualmente.

Continuando dicha reflexión Jaspers señalará en "Rencontres Internationales de Génève" de 194916, que vivimos con la era tecnológica, en un enorme aparato técnico-político, indispensable para el mantenimiento y el bienestar de la civilización de masas y, al mismo tiempo, pleno de potencialidades destructivas en ciernes para la humanidad del hombre. Combinar el cumplimiento de los reclamos de las masas con y mediante el dominio tecnológico, en condiciones que aseguren la libertad pública del espíritu, es la única forma de salvar nuestra civilización y nuestra humanidad.

De la misma forma, J. Huizinga ${ }^{17}$ y Karl Manheim ${ }^{18}$, al comienzo de la dominación nazi, se dan cuenta de que el resultado de la revoIución científico-tecnológica, con todo su énfasis en la racionalidad y el control de la naturaleza por el hombre, fue la irrupción de la civilización de masas, llena de empujes y tendencias irracionales alarmantes que harian peligrar la supervivencia del humanismo. Mientras que, sin embargo, Manheim cree en la posibilidad de disciplinar las colosales fuerzas nuevas mediante la planificación democrática $^{19}$, Huizinga es mucho más pesimista. Ortega y Gasset ${ }^{20}$ piensa que el hombre-masa -entendido como un tipo psico-cultural, independientemente de consideraciones de clase social- representa una forma degradada de humanidad, que expresa la masificación de la vida moderna. Spengler, por su parte, piensa que Occidente ha perdido su fuerza creativa cultural y que ha entrado en una fase de civilización mecánica. Sus tenues esperanzas radican en una posible catarsis espiritual, en la que la juventud tendría que representar un papel decisivo, volviendo a introducir la disciplina y los valores del humanismo en el sistema de la civilización de masas.

Reflejando, en la secuela de la segunda guerra mundial, la misma línea de preocupaciones, Hans Freyer ${ }^{21}$ observa cómo el sistema de

${ }^{10}$ Publicado en Balance and Perspective, edic. alemana R. Piper \& Co. Verlar, Munchen, 1951, trad. española Revista de Occidente, Madrid, 1951.

${ }^{1 " C f}$. J. Huizinga, Amid the Shadows of Tomorrow, edic. original alemana de 1935 .

${ }^{18} \mathrm{Cf}, \mathrm{K}$, Manheim, Freedom and Social Planning, edic. alemana original publicada en Holanda, de 1935.

${ }^{10} \mathrm{Ver}$ su Diagnosis of our Time, edic. inglesa original de 1943 y Freedom, Power and Democratic Planning, edic. orig. inglesa de 1950.

${ }^{20} \mathrm{Cf}$. José Ortega y Gasset, La Rebelión de las Masas, edic. original 1930, vol. IV de Obras Completas, Revista de Occidente, Madrid, 1947.

${ }^{21} \mathrm{Cf}$. Hans Freyer, Theory of the Present Time, edic. original alemana 1955. trad. española por Fondo de Cultura Económica, Breviarios, Mréxico, 1958. 
civilización de masas, orientada hacia la consumación de la historia por la tecnología, ocasiona la fragmentación del hombre moderno en papeles distintos y separaclos -elector, consumidor; contribuyente impositivo, etc., $-\mathrm{y}$ lo aliena de sí mismo.

El mismo pesimismo profundo acerca de las condiciones actuales y las perspectivas futuras cle la Civilización Occidental, que ahora abarca el mundo entero, se encontrará en las opiniones de Sorokin sobre nuestro tiempo22. Sin embargo, Sorokin, según su teoría cíclica general de la historia, piensa que dicha crisis expresa la ya avanzacla descomposición del período sensato tardío de Occidente y prevé un no lenajo re-nacimiento socio-cultural, con el próximo surgimiento de una nueva cultura integracla.

Analistas no menos agudos pero más cerca de nuestra época, no obstante, presentan un punto de vista menos necesariamente catastrófico de sus tendencias. Kenneth Boulding ${ }^{23}$ sostiene que, en última instancia, la humanidad ha pasado por clos grandes transiciones. La primera fue la transición hacia la civilización, del salvajismo paleolítico a la barbarie neolítica. La segunda, que se desenvuelve actualmente, es la transición de la civilización a la post-civilización, es decir, de un período de adaptación a la naturaleza, a un período de control social y cle autocontrol. Dicha transición, sin embargo, dista mucho de ser automática y no es difícil que la humanidad se pierda, clesembocando en trampas fatales. Ellas son, 1) la guerra, 2) el fracaso del intento de promover el clesarrollo general del munclo, 3) el crecimiento demográfico incontrolado y 4) la trampa de la entropía. Esta última puede presentar un carácter cultural, en el caso de una pérclida de potencial creativo, o un carácter natural, en el caso de merma de la riqueza mineral de la tiema.

Consideranclo los mismos problemas en una perspectiva más amplia, Erich Kahler recalca el hecho de que Occidente ha llevado a la sumaniclad al borde de la auto-aniquilación o la auto-regulación'24. En su obra más reciente observa que la evolución cultural, continuando la evolución biológica por otros medios, empuja ahora el proceso evolutivo en dirección socio-colectiva, y no ya en dirección individual. En las palabras de Kahler: "la tremenda expansión reciente de los alcances del hombre, y la incorporación de contenidos mundiales en la conciencia humana, se aplica al hombre como un todo, y no ya al hombre individual"'25.

${ }^{29}$ Cf. Pitirim Sorokin, The Crisis of Our Age, E. P. Dutton, N. Y., I9t1 y The Basic Trends of our Time - College \& University Press, New Haven, 1964. ${ }^{2} \mathrm{Cf}$. Kenneth Boulding, The Meaning of the 20th Century, Harper Colophon Book, N. Y., 1964.

${ }^{24 C f .}$ Erich Kahler, The Meaning of History, Braziller, N. Y., 1964.

${ }^{2} \mathrm{C}$. Erich Kahler, Out of the Labyvinth, p. 16, Braziller, N. Y., 1967. 
Si ahora giramos el foco de atención de los aspectos socio-culturales del mundo contemporáneo hacia su parámetro físico veremos, entre los estudiosos de los recursos naturales y el ecosistema humano, un apoyo sostenido de la alarma del CoR concerniente al rápido crecimiento del desequilibrio entre la presión clemográfica real y la capacidad del ecosistema. Limitaré mis indicaciones a ciertos items cruciales tales como alimentos, materias primas y algunas formas globales de desequilibrio.

El problema de calcular la capacidacl potencial del mundo para la producción de alimentos comprende muchos aspectos complejos, descle la estimación, en el actual nivel tecnológico, de las posibilidades extractivas óptimas de elementos nutritivos de sus posibles fuentes, hasta la consideración de los varios factores que interfieren en los supuestos anteriores, desde las innovaciones tecnológicas hasta los cambios institucionales y organizativos. Teniendo en cuenta todos los aspectos, los expertos tienden a coincidir en que un aumento substancial es factible fácilmente, pero que no debe esperarse más de 4 a 5 veces la producción actual ${ }^{26}$, o cuando más, a largo plazo, 9 veces más ${ }^{2 \pi}$. Un cletalle interesante acerca de la producción potencial cle alimentos es la reducción, a niveles mucho más modestos, de los cálculos anteriores en relación con las posibilidades de obtención de alimentos del mar². Una población teórica máxima de 30 billones correspondería al límite superior de capacidad cle producción alimenticia en el mundo 20 . Los mismos expertos, sin embargo, al transferir ese máximo teórico a un nivel cle posibilidades prácticas, calculan en 10 billones el "máximo razonable de población, en un mundo que seguirá necesitando administración intensiva"30.

Las perspectivas en relación con la disponibilidad mundial de materias primas tampoco son compatibles, según los expertos, con un prolongado crecimiento demográfico, particularmente si tal creci-

20Afirmación de James Bonner en The Next Ninety Years - Proceedings of a Conference Held at the California Instilute of Tecnology, marzo de 1967, p. 31 sig. California Institute of Technology, Pasadena, 1967.

:TResources of Man, por la Comisión de Recursos y el Hombre (Committee on Resources and Man), National Academy of Sciences and National Research Council, p. 5, W. H. Freeman \& Co., San Francisco, 1969.

${ }^{25} \mathrm{CE}$. William E. Ricker, "Food From the Sea", en Resources and Man, op. cit., pp. 87-108. Dados los diversos niveles tróficos, desde la fotosintesis de plancton hasta los animales marinos mayores, un promedio de 130.000 toneladas de plancton produce solamente 33 toneladas de peces comestibles en promedio.

${ }^{20} \mathrm{Cf}$. Introducción a Resources and $\mathrm{Man}$, op. cit., p. 5.

soldem, ibid. 
miento debe estar asociado, como se desea universalmente, con un desarrollo económico continuo y generalizado. Como se ha señalado. en los estudios del CoR, el efecto combinado del crecimiento demográfico, el desarrollo tecnológico y el aumento del PBN per cápita, aumenta a una velocidad fantástica la presión demográfica real sobre los recursos. Los cálculos de John McHale, en forma similar. a los de Peccei, indican que, mientras en 1880 , se consumía 50 :tonelàdas de materia prima poi persona, en 1966 esa cifra subió a 300 toneladas $^{31}$. Suponiendo una población mundial de 7.5 billones en el año 2000, Harrison Brown ${ }^{32}$ estima que, para darle a la gente' el estándar de vida que ahora se disfruta en los Estados Unidos, sería necesario extraer de la tierra, entre otros, los siguientes materiales anualmente: 50 billones de toneladas de hierro; I billón de cobre; I billón de plomo; 600 millones de zinc; 100 millones de aluminio. Dichos montos de actividad minera representan cientos de veces el promedio actual y "virtualmente agotarían los recursos minerales de alto grado y requerirían que viviésemos de las substancias más pobres de la tierra: las aguas del mar y la roca común".

Mientras que las materias primas y alimenticias presentan límites específicos más crudamente determinables para el crecimiento de la población y el aumento de la presión demográfica real, los ecólogos están de acuerdo también con las afirmaciones del CoR en relación con la corriente actual de desequilibrar el medio ambiente natural general del hombre. En general, observa Marston Bates ${ }^{33}$, el ecosistema humano ha sido perturbado por el hombre sin el conocimiento adecuado. "La cuestión que se cierne amenazadoramente sobre el futuro de nuestra especie es la siguiente: cadquiriremos sabiduría para utilizar este conocimiento y para generar el nuevo conocimiento requerido para evaluar y enfrentar las consecuencias ambientales del avance tecnológico?

Además de los problemas culturales y físicos que surgen de la continua. expansión exponencial de población, tecnología y consumo, otro aspecto perturbador importante del mundo contemporáneo es la velocidad continuamente ascendente en que se operan los cambios. La constante aceleración de la transitoriedad, como lo ha observado acertadamente Alvin Toffler -a pesar del carácter discutible de varias de sus afirmaciones- está creando un "shock futuro" psicológicamente destructivo en sectores crecientemente más

${ }^{31} \mathrm{Cf}$. John McHale, The Ecological Context, p. 9, Braziller, N. Y., 1969.

${ }^{32}$ Cf. Harrison Brown, The Next Ninety Years, op. cit., pp. 16-17. cit., p. 29. 
ámplios de la sociedad contemporánea, capaz de sobrepasar los límites de la adaptabiliclad natural del hombre ${ }^{34}$.

En conclusión, y considerando los diversos aspectos que se han senalado en el tema anterior y el presente, se pueden hacer las siguientes generalizaciones básicas:

1. El crecimiento exponencial de la tecnología y la población llevó a un cambio cualitativo en la civilización occidental, que ahora cubre todo el orbe, dándole a nuestro tiempo caracteres históricos únicos, como lo observan Karl Jaspers y otros;

2. Estos cambios expresan, por un lado, una continuación de la evolución cultural del hombre, desde sus comienzos paleolíticos, y parecen encaminar el proceso evolutivo en la dirección sociocolectiva del hombre como un todo, más bien que como individuo, como lo apunta Kahler. Por otra parte hay señales manifiestas de descomposición, en muchos niveles de la vida socio-cultural de las sociedades contemporáneas. Esto parece indicar que estamos viviendo no sólo como cultura sino como especie en el umbral de la auto-organización o auto-destrucción social.

3. La extensión, en número, complejidad y velocidad, de los factores relevantes que afectan a la humanidad contemporánea como sus relaciones con su ecosistema natural, tales como niveles de población, tecnología, y producto per cápita, como también el carácter de los valores culturales y estilos de vida, han llegado a un punto más allá del cual los procesos noregulados, las velocidades de crecimiento y las decisiones fragmentadas ya no son compatibles con la supervivencia de la especie a largo plazo.

\section{I.OS ASPECTOS POLITICOS}

LAS CUESTIONES BÁSICAS QUE ESTAN EN JUEGO

Los breves comentarios, presentados en la parte anterior de este trabajo, sobre las primeras dos áreas principales de cuestiones enumeradas en su comienzo, proporcionan el necesario marco de referencia para el análisis apretado, en esta tercera y última parte del presente estudio, de las dos cuestiones que restan: los problemas del equilibrio ecológico mundial en función a) del sistema internacional y b) del desarrollo del Tercer Mundo. Estos dos órdenes de

${ }^{34}$ Cf. Alvin Toffler, Future Shock, Random House, N. Y., 1970. 
cuestiones conciernen a los aspectos políticos de los problemas ecológicos del mundo y deben tratarse conjuntamente.

EI primero y más amplio comprende, de un modo general, al segundo, ya que el Tercer Mundo - por el que se entiende las naciones infradesarrolladas en comparación con el Primer Mundo de naciones capitalistas desarrolladas y el Segundo Mrundo de maciones socialistas desarrolladas- no es más que una parte, la más grande y la más pobre, del sistema internacional. La segunda área de cuestiones, sin embargo, no puede reducirse simplemente a las relaciones internacionales de los países subdesarrollados con las partes desarrolladas del mundo y debe verse en función de las necesidades específicas del desarrollo social ${ }^{3 \breve{5}}$, confrontadas, en lo que hace a este trabajo, con las necesidades del equilibrio ecológico mundial.

Como se ha indicado en las conclusiones de la Parte II que antecede, nuestro tiempo está ubicado en un período histórico único, no sólo en el sentido en que todo período histórico es específico e irreductible a cualquier otro período, sino en el sentido de que por primera vez, desde la existencia del hombre, su supervivencia como especie dependerá de la aclecuada auto-regulación colectiva, comprendiendo, notablemente, la regulación mundial de la población, de la tecnología y los estilos de vida y de los recursos naturales.

Considerado desde un punto de vista político amplio este imperativo general de la auto-regulación colectiva implica esencialmente Ia prevención de tres posibles resultados suicidas de la sociedad contemporánea: 1) el holocausto atómico, 2) la ruina del ecosistema y 3) la desintegración socio-cultural de la sociedad30.

La prevención de estos tres resultados suicidas implica la necesidad de algún nuevo orden mundial efectivo y significativo, capaz de proporcionar los controles negativos requeridos para impedir el desencadenamiento de guerras atómicas en gran escala $y$, lo que probablemente resulte más difícil aún, capaz de proporcionar los controles positivos para la administración mundial del sistema natural ecológico y para una re-integración socio-cultural amplia, en los niveles internacional y nacional, de la sociedad contemporánea.

Las dificultades de organización de un sistema de orden mundial capaz de suministrar los controles negativos necesarios para impedir las guerras atómicas en gran escala han sido discutidas ampliamente por un gran número de escritores y yo mismo he tratado el tema

\footnotetext{
${ }^{35} \mathrm{Cf}$. entre otros Louis Irving Horowitz, Three Worlds of Development, Oxford Univ. Press, N. Y., 1966.

${ }^{30} \mathrm{Cf}$. Sir MacFarlane Burnet, afirmación intimamente relacionada: "Hay tres imperativos: reducir la guerra al mínimo; estabilizar la población humaná; e impedir la destrucción progresiva de los recursos no recuperables de la tierra", citado en Resources and Man, op. cit., p. 1.
} 
en otra parte ${ }^{3 \tau}$. No hay mucha duda con respecto al hecho de que lo que se necesita para la administración mundial del ecosistema natural y para la re-integración socio-cultural internacional y nacional de la sociedad contemporánea implica un tipo de orden mundial aún más difícil de obtener. A pesar de ello, parece aún menos dudoso que la alternativa de no conseguir tal orden mundial a tiempo será la auto-destrucción de la especie. Esto es precisamente Io que quiere decir el Club de Roma al referirse al dilema actual de la humanidad.

Como se dijo anteriormente se hará un intento, en esta parte final del trabajo, tomando en cuenta los comentarios anteriores sobre los estudios del Club de Roma y sus conclusiones y las cuestiones respectivas, de analizar brevemente sus aspectos políticos. Con tal fin procuraré adelantar algunas indicaciones muy rápidas acerca del sistema internacional actual y algunos de los problemas críticos ecológicamente relacionados que están en juego, con el fin de intentar una breve valoración de las tendencias y posibilidades de dicho sistema internacional, en su confrontación con la crisis ecológica. Finalmente, algunas conclusiones en términos de las necesiclades. en cuanto a conocimiento, política y acción que resultan de la situación ecológica actual y sus cursos alternativos probables.

\section{EL SISTEMA INTERNAGIONAL ACTUAL}

La comprensión del sistema internacional actual ha sido durante mucho tiempo perturbada por los efectos combinados de los prejuicios ideológicos y la persistencia de los puntos de vista tradicionales en cuanto a política internacional. No sería posible aquí iniciar una discusión sistemática del asunto. Basta con recordar el hecho de que el imponente prestigio del sistema internacional centrado en la Europa del siglo xIx y primera parte del $\mathrm{xx}$, y la abundante literatura en torno a su mecanismo de equilibrio de poder, ha sobrevivido, como representación mental, mucho después de que las relaciones internacionales habian cambiado profundamente su carácter. Al mismo tiempo el maniqueísmo ideológico de la guerra fría manturo al mundo envuelto en las cruzadas anti-comunista $y$ anti-capitalista, lanzadas respectivamente por cada una de las dos. superpotencias, impicliendo que los estudiosos y estadistas por igual pudieran darse cuenta del hecho de que lo que realmente estaba en juego era la formación de nuevos grandes sistemas imperialistas.

Acontecimientos recientes, tanto en esferas académicas como en:

${ }^{37}$ Cf. Helio Jaguaribe, "World, Order, Rationality and Socioeconomic Development" en Daedalus, vol. 95, N. 2, primavera de 1966, pp. 207-226. 
las de la política práctica, han dado lugar a un punto de vista enteramente nuevo sobre el sistema internacional. Como to ha aclarado uno de los más brillantes analistas nuevos, Georges Liska ${ }^{38}$, el nuevo sistema internacional es, esencialmente, un sistema inter-imperial. Algo más precisamente, se lo puede definir como un sistema bipolar, unifocal y bisegmentado, evolucionando inciertamente hacia la multipolaridad ${ }^{39}$. Unicamente las dos superpotencias disfrutan de condiciones de primacía, si bien los Estados Unidos ejercen primacía general, lo que constituye el foco central del sistema internacional, obligando a la Unión Soviética, si bien cada vez menos, a la primacía regional. La bisegmentación del sistema internacional, por otra parte, expresa la división del mundo subdesarrollado versus el desarrollado, por debajo de la alineación imperial.

Dicho sistema ofrece, en última instancia, una estratificación a cuatro niveles. El nivel más elevado está ocupado por la potencia que actualmente disfruta de primacía general, los Estados Unidos. El segundo nivel corresponde a la primacía regional. Actualmente lo ocupa la Unión Soviética, a la vez que se esfuerza por alcanzar primacía en condominio con los EE. UU. China, como nueva potencia imperial que surge, se acerca a la primacía regional, y el sistema europeo, si realmente desemboca en una comunidad económico-política más sólida y firme, puede saltar a dicho nivel. El tercer nivel de estratificación internacional corresponde al nivel de autonomía: la situación de las potencias que no disponen de suficiente capacidad nuclear de respuesta y cuasi auto-suficiencia, pero que tienen condiciones para hacer muy costosa cualquier agresión externa unilateral y de beneficiarse de un amplio margen de elección independiente. Tal es el caso actual de China y, en el sistema europeo, el de sus países más grandes, comó también el caso del Japón. Tal puede ser, en las próximas décadas, el caso de América Latina y actualmente de la India, del Mundo Arabe-Africano, y tal vez de Indonesia. El cuarto y último nivel de estratificación internacional es el de la dependencia y corresponde a países que no disfrutan de las condiciones anteriormente indicadas, es decir, el resto del mundo ${ }^{40}$.

${ }^{38} \mathrm{Cf}$. George Liska, Imperial America, 1967, Alliances and the Third World, 1968 and Ward and Order, 1968, todos publicados por la John Hopkins Press, Baltimore.

${ }^{39}$ Ver Liska, Imperial America, op. cit., p. -36 y Alliances and the Third World, op. cit., p. 8.

${ }^{10}$ Calificaciones importantes, que, no pueden aclararse aquí, deberian introducirse con respecto a la posición de países, incluidos en "el resto del mundo", como Canadá y Australia, muy relacionadas con los Estados Unidos e Inglaterra, como también en relación con las posibilidades individuales de Brasil $y$, hasta cierto punto, México y Argentina, y aun, eventualmente, Pakistán. 
Este nuevo sistema inter-imperial es un sistema dinámico y complejo, en el que las posiciones de los actores, en el curso del tiempo, son afectadas por una variedad de factores y con ello sus interrelaciones que presentan también un esquema complejo cle cooperación-competente, dominio-clependencia, áreas de influencia, niveles $y$ reciprocidad de inter-influencia, etc. Considerando en forma. simplificada dicho esquema complejo, tres ordenes de tensiones $y$ conflictos resultan discernibles: 1) el inter-imperial, 2) el intraimperial y 3) el que concierne a los valores de interés de los paises, clases, sectores y grupos desarrollados vs. los subdesarrollados.

Mientras que las tensiones inter-imperiales, en las dos décadas que siguieron a la II Guerra Mundial, han sido más visibles y tendieron a atraer más la atención, los conflictos intra-imperiales entre las superpotencias y sus países dependientes van aumentando en importancia, como así también los conflictos de valores de interés entre los países y dentro de los mismos. En paridad de condiciones, un cambio de énfasis de los inter-imperiales a los intra-imperiales tiende a reducir los riesgos de las guerras atómicas en gran escala, pero también a aumentar las dificultades para una adecuada administración mundial del ecosistema. Efectos similares resultan de los aumentos de conflictos cle valores de interés entre los países y dentro de los mismos.

\section{ALGUNOS PROBLENAS GRÍTICOS}

No sería posible, dado el carácter reclucido del presente trabajo, analizar sistemáticamente los principales problemas críticos que afectan al actual sistema internacional, en función de los problemas ecológicos de nuestro tiempo. Mencionaré simplemente, como ilustración, tres clases particularmente críticas de problemas.

El primero se refiere al mantenimiento intra-imperial, y a los límites de la soberanía y la jurisdicción territorial de los países dependientes. A diferencia de los Imperios anteriores, que tendían a formalizar la relación de dependencia entre el centro metropolitano y su periferia subordinada (sátrapas persas, provincias romanas, colonias británicas) los Imperios contemporáneos mantienen la ficción legal de la independencia de sus satélites. Tanto en el caso norteamericano como en el soviético dicha ficción legal está profundamente enraizada en la ideología legitinizante del centro metropolitano: el liberalismo contractualista norteamericano (que implica una libre asociación de países libres), la igualdad dialéctica soviética (que implica una igualdad de status y asociación basada en la eliminación de la propiedad privada y la explotación social).

Confrontada, sin embargo, con una tendencia hacia una general 
disminución de los recursos naturales, una demanda siempre creciente de nuevas tierras y además una siempre creciente necesidad de utilizar nuevas fuentes potenciales de suministro de materiales, tal como los mares, los Centros Imperiales están descubriendo que la forma convencional de presionar a sus satélites para ganar acceso adecuado a dichos elementos se hace cada vez más insuficiente. Esto es lo que está en la raíz de las crecientes tensiones intra-imperiales mencionadas anteriormente.

Una contradicción creciente, por lo tanto, es predecible entre los principios ideológicos y reglas legales actuales y las prácticas de los Centros Imperiales y sus necesidades vitales, en relación con dichos elementos o facilidades. En la medida en que la tendencia hacia la merma de dichas facilidades no sea contenida, la contradicción entre principios e intereses tendrá que resolverse a expensas de uno de estos polos. No cabe la menor duda, como lo enseña la práctica histórica, que tal contradicción se resolverá a expensas de los principios. En consecuencia, las relaciones intra-imperiales tenderán a volverse menos legítimas en forma creciente, en términos de valores, y menos equilibradas, en términos de intereses, agravando la extensión y la intensidad de los conflictos de valores de interés entre los países y dentro de los mismos.

La segunda clase crítica de problemas que requiere una referencia concierne a la propensión ideológica, tanto de izquierda como de derecha, en países subdesarrollados, a considerar abierta o secretamente, que los aumentos grandes de población están rindiendo aumentos aproxinadamente equivalentes de fuerza. La bomba de población, para usar el título del "best seller" del doctor Ehrlich, ${ }^{41}$ tiende realmente a ser considerada como un arma política tanto por los revolucionarios maoístas como por los estadistas de derecha. Los primeros piensan que fuertes incrementos demográficos no acompañados por una colrespondiente expansión de las oportuniclades de trabajo y servicios sociales, acumularán una presión revolucionaria que en cierto momento hará explotar los regímenes conservadores. Los últimos piensan que su capacidad de negociación con su propio Centro Imperial -al cual adhieren básicamente pero dèl cual buscan obtener más independencia de maniobra- aumentará proporcionalmente al peso demográfico de sus países.

Limitando la discusión del asunto a unos pocos comentarios, permítaseme comenzar por admitir là existencia de una obvia correspondencia potencial entre población y fuerza política. Siendo otras variables iguales, un país de 100 millones de habitantes es sustancialmente más fuerte -si bien no en una proporción lineal- que

${ }^{41}$ Cf. Paul R. Ehrlich, The Population Bomb, Ballantine Books, N. Y., 12th. impresión, 1970, primera impresión 1968. 
Helio Jaguaribe / El equilibrio ecológico mundial y los países subdesarrollados

un país de 10 a 50 millones de habitantes. Todo el problema consiste en la distinción entre la verdadera fuerza de población y la población numérica o bruta. La verdadera fuerza de población no está dada por gente, aisladamente, sino, fundamentalmente, por la combinación de la población con su grado de l) integración sociocultural, 2) movilización ideológica, 3) organización efectiva y 4) equipo económico-tecnológico.

La fuerza de los Estados Unidos, hasta hace poco, fue el resultado del muy alto grado en que poseía los cuatro factores arriba mencionados, para una población numérica, si no pequeña, en términos internacionales sustancialmente más pequeña que la de China o de la India. Fue el hecho de los conflictos de valores-interés (resultantes de la guerra de Vietnam, de los movimientos de derechos civiles, de los nuevos valores culturales de la juventud y de otras circunstancias, han disminuido significativamente la extensión de la integración socio-cultural norteamericana, la movilización ideológica y, como resultado, parte de su nivel organizativo), lo que hizo que los Estados Unidos estén actualmente siendo privados, para muchos propósitos, de una gran parte de su fuerza anterior, aun cuando, entretanto, la población norteamericana haya aumentado sensiblemente.

Si, por otra parte, se compara dos países muy poblados, la China y la India, ambos igualmente pobres en equipo económico-tecnológico, la neta superioridad de China, tanto en términos de su status internacional como en términos de su tasa de desarrollo, corresponde claramente a su nivel superior de integración socio-cultural, movilización ideológica y organización efectiva. Comparaciones similares señalarían también la neta superioridad de Israel, con su pequeña población, frente a los fuertemente poblados pero pobremente movilizados y organizados países árabes.

Las consideraciones citadas son suficientes para revelar los crasos errores contenidos en una pura teoría de fuerza de la población bruta. Esto no significa, sin embargo, que todas las formas de control de población estén ocasionando un incremento en la verdadera fuerza de población. Como se lo indicó previamente la verdadera fuerza de población resulta principalmente de la combinación de la población bruta con los cuatro factores arriba indicados. Más a menudo de lo que se piensa, grandes países subdesarrollados, tales como Brasil o México, incrementarían su verdadera fuerza de población, siendo otros factores iguales, con sólo tener proporcionés más pequeñas de crecimiento demográfico y permitiendo una mejor distribución de facilidades educacionales y sanitarias, así como también de factores producticos, para su población. Todo el énfasis tiene que ser puesto, sin embargo, en la consideración de que un creci- 
miento demográfico menor (problemas ecológicos aparte) no es, en sí mismo, un paso automático hacia un rápido desarrollo social, sino sólo una condición que hace más fácil la adopción de mejores políticas de distribución de los recursos disponibles.

Si se agregan consideraciones ecológicas al presente trabajo, la importancia relativa y absoluta de reducir el crecimiento demográfico es sustancialmente realzada. Sólo haré dos breves comentarios sobre el tema. El primero concierne a la diferencia de la relación entre población por una parte, y recursos expandibles y no expanclibles, por la otra. Cuando los recursos son expandibles, tal como muchos recursos renovables, menos población hace más fácil una mejor colocación, pero si tales condiciones más fáciles no se usan, la reducción de población no mejorará su régimen de distribución. Es claro que, si los recursos correspondientes no son expandibles, $y$ otros no-renovables, cualquier consumidor adicional, cualquiera fuera su parte, está disminuyendo la obtenibilidad total de tales recursos.

El segundo comentario que deseo hacer está relacionado con Ia cuestión ya discutida de la soberanía legal, en condiciones intra-imperiales. Dada una situación de dificultades ecológicas y una tendencia creciente hacia la escasez de materiales, no puede haber duda que cuanto más grande sea una población dependiente y cuanto más bajo en ella sea el nivel de los cuatro factores ya mencionados, tanto más estará expuesta a las prácticas veraces de su Centro Imperial y tanto más bajos serán su estándar de vida y sus posibiliclades de supervivencia.

Permítaseme ahora presentar brevemente la tercera y última clase de problemas críticos que estoy tratando de discutir: Ios conflictos de valores de interés que surgen en última instancia de condiciones sustancialmente desparejas de desarrollo entre paises, estratos $y$ grupos. Tal falta de uniformidad se presenta, en nuestra época, en tres formas principales:

1) el contraste entre el alto nivel de desarvollo de muchos paises del hemisferio Norte y el muy bajo de la mayor parte del hemisferio Sur;

2) la diferencia de auto-determinación entre los Centros Imperiales y las periferias dependientes;

3) la diferencia de oportunidades $y$ de niveles de ingresos, cultura, poder y prestigio entre clases sociales altas y bajas, en todo el mundo.

Esta última, en algunos casos - como tiende a ocurrir en Ios centros metropolitanos - se reduce a una diferencia más sutil pero no menos efectiva entre las clases sociales, las oportunidades para ob- 
tener $y$ disfrutar cultura, poder e influencia, aunque la diferencia no sea tan notable al nivel corriente del consumo económico personal.

Las perturbaciones socio-económicas de las sociedades contemporáneas no son ciertamente reclucibles a las tres formas de falta de uniformidad arriba indicadas. Como se especificó brevemente en la Parte II de este trabajo estamos vivienclo la gran crisis de la cultura occidental, crisis que involucra el agotamiento de sus mitos religiosos y un profundo reto a su ética humanística. No puede haber dudas, sin embargo, cualesquiera sean las otras variables intervinientes, de que la legitimiclad cle nuestros estándares éticos está decisivamente afectada por la contradicción, a los niveles internacionales y nacionales, entre los principios y las prácticas cle la igualdad y la libertad. $Y$ las tres formas arriba indicadas de la falta de uniformidad se encuentran en el fonclo de tales contradicciones.

Analizando, de forma muy sucinta, $y^{t}$ con perspectiva ecológica, las consecuencias cle la triple falta de uniformidad ya citacla (la desigualdad de desarrollo entre las naciones, cle auto-determinación de las periferias y entre las clases sociales), otro conjunto triple de efectos negativos pueden señalarse.

El primer efecto negativo a mencionar concierne al hecho de que, en el transcurso del tiempo, un incremento inevitable del conocimiento, por un número cada vez mayor de pueblos y gente explotados, de su condición de explotación, reduce correspondientemente su lealtad espontánea a los sistemas a los que están someticlos, creando la necesidad de un uso incrementado de la coerción, con una proporción necesariamente decreciente de los logros del sistema y del margen de beneficios que puede producir. Ese efecto, incluyendo sus formas reflejas y derivativas -a menudo en sí mismas arbitrarias- puede detectarse, de muchos modos, en la experiencia general en incremento, que las instituciones, descle los órganos del Estado, hasta las empresas y las familias, no están trabajando bien. $\mathrm{Si}$ se agregan consideraciones cle escasez ecológica a este cuadro, la proporción resultante de mal funcionamiento se aumenta correlativamente.

El segundo efecto negativo a enfatizar corresponde a la contradicción en aumento, en términos puramente utilitarios, entre una civilización tecnológica de producción y consumo masivos y el mantenimiento, de varias maneras, a los niveles internacional y nacional, de prácticas discriminativas imperantes clescle los tiempos preindustriales que implican un juego "suma-cero" a costa cle los "de afuera." En cuanto que los proletariados externos explotables, entendidos tanto en términos internacionales como nacionales, han desapareciclo, debido al carácter totalizaclor de la civilización contemporánea, las reglas "suma-cero" se anulan a sí mismas, tanto descle 
el punto de vista del sistema global -es decir, la sociedad contemporánea como un todo- como descle el punto de vista de los cliversos grupos dirigentes en cuestión, que están autodestruyendo sus propias clientelas y áreas de acción. $Y$ agregar consideraciones de escasez ecológica a ese cuadro lo convierte en un proceso loco, porque, a su carácter cualitativo auto-anulador, se agrega una propensión a su agotamiento físico irreversible.

$Y$ "last but not least", el tercer efecto negativo a señalar está relacionado con el deterioro moral resultante, en todos los niveles, de la crisis de legitimidad ya mencionada que nuestros estándares éticos están sufriendo, del hecho mismo de su violación rutinaria por la triple falta de uniformidad ya inclicada.

\section{TENDENGIAS $Y$ PERSPEGTIVAS}

Como se ha comentado ya en este estudio, la sociedad contemporánea se encuentra en el umbral de la auto-regulación o la autodestrucción. El resultado que se obtenga estará determinado por el manejo efectivo o inadecuado de las tres cuestiones básicas con las que se confronta nuestro tiempo: 1) la forma de impedir la guerra atómica en gran escala; 2) la regulación apropiada y oportuna del ecosistema natural; 3) la re-integración socio-cultural amplia, en los niveles internacional y nacional, de la sociedad contemporánea.

El riesgo de un holocausto atómico, inmediatamente realizable toda vez que la capacidad de responder con muerte masiva está al alcance de cada una de las dos superpotencias, llevó al desarrollo de reglas implícitas y explícitas de coexistencia que han permitido, hasta ahora, mantener la paz básica. El nivel institucional de tal coexistencia es extremadamente precario y naturalmente requiere mejoras sustanciales, si se ha de preservar esa paz básica por mucho tiempo. A pesar de tal precariedad, el alto grado de compenetración, por parte de ambas superpotencias, de los riesgos que acarrea y la cautela con que han impedido, hasta ahora, las confrontaciones irreversibles, han ofrecido un cuadro más promisorio que lo que hombres inteligentes, como Bertrand Russell, estaban dispuestos a creer en los primeros años de la década del sesenta ${ }^{42}$.

La aparición de China como potencia internacional de importancia, capaz de alcanzar primacía regional en el curso de dos décadas, con la correspondiente elevada capacidad nuclear, introducirá, sin embargo, un importante factor de complicación en el sistema inter-imperial. El juego atómico tri-partito, en lugar del actual juego bi-partito, aumentará considerablemente las complicaciones del

${ }^{42}$ Cf. Bertrand Russell, Has Man a Future? Penguin Books, Baltimore, 1961. 
proceso de preservación de la paz, requiriendo al mismo tiempo. mecanismos institucionales más eficientes. En la medicla que en que continúe la difusión atómica, aumentarán las complicaciones. para mantener la paz, la que se hará imposible sin una institución internacional formal apropiada y un compromiso mundial firme que la apoye.

La crisis ecológica ha llevado más tiempo para que los expertos y los estadistas se percataran de ella, pero ahora parece estar adquirienclo rápidamente comprensión total ${ }^{43}$. Todavía vivimos una seria clesproporción entre el reconocimiento general, que comienza a manifestarse, de que algo sumamente serio anda mal en el ecosistema natural del hombre, y los alcances y el ritmo de dicho deterioro. Sin embargo, dado el reconocimiento que ya se ha alcanzado acerca de la crisis ambiental, es probable que la década actual y las posteriores de este siglo dedicarán máxima preocupación y esfuerzo para resolver el problema.

Gon todo, a diferencia de lo que está ocurriendo en relación con los riesgos del holocausto atómico y el suicidio ecológico, el riesgo de una desintegración socio-cultural de la sociedad no se comprencle ni se toma en cuenta suficientemente. En este sentido nos enfrentamos con un orden de problemas infinitamente más complejo, que expresa, por una parte, las dificultades combinadas de los otros dos órdenes y, por otra, trascienclen el parámetro del mero tratamiento científico pues incluye todas las dimensiones del problema humano. Reduciendo un asunto tan complejo a su forma más simple creo que pueden destacarse los siguientes aspectos:

1) La crisis socio-cultural de la sociedad contemporánea, en sus niveles internacionales y nacionales, expresa la crisis de las creencias básicas de la cultura occidental universalizada, y en ese sentido es un problema religioso, ético y filosófico que trasciende sus condicionamientos sociales;

2) A pesar de la observación anterior, la desintegración sociocultural de la sociedad contemporánea está directamente vinculada con el triple desnivel anteriormente inclicado en este estudio: I) el contraste del desarrollo de los hemisferios Norte y Sur; II) el contraste de la auto-determinación de los Centros Imperiales y las Periferias Dependientes y III) las diferencias de oportunidad $y$ de estándar de vida de las clases sociales;

3) Mientras se ha reconociclo la triple falta de uniformidad ya citada, su enfoque ha sido doblemente insuficiente. Insuficiente en calidad porque el subclesarrollo y la marginación social se han tratado como simples casos de subcapitalización, cuando son en realidad

${ }^{4} \mathrm{Cf}$. The Time, edición de febrero 2, 1970. 
expresiones socio-culturales y socio-políticas de igualdad negada y de libertad suprimidat4. Insuficiente en cantidad porque, aun cuando fueran tratados como casos de subcapitalización, los fondos totales, a niveles internacionales y nacionales, han estado manifiesta y sustancialmente por debajo de los mínimos requerimientos críticos para lograr cambios que se auto-mantengan ${ }^{45}$.

Dada la situación amiba desarrollacla brevemente, parece que un análisis prospectivo cle las probables tendencias de desarrollo de tal situación conduciría a las tres siguientes alternativas básicas:

1. Una alternativa "central", suponienclo que una comprensión ya eviclenciada de los riesgos atómicos y ecológicos generaría políticas apropiaclas para su prevención;

2. Una alternativa agregada "desviada", supuestos a) ya sea un fracaso de comprensión por grupos responsables, que les impida formular políticas apropiadas para evitar los clos riesgos arriba mencionaclos, o b) un fracaso en la implementación de políticas atómicas y ecológicas, en principio apropiadas, conducentes a errar sus propósitos expresos;

3. Una alternativa cle efectos "socialmente perversos", resultantes del hecho de que la alternativa "central", si bien correctamente acloptada e implementacla, en tanto estarían involucrados los riesgos atómicos y ecológicos, debiclo a su fracaso para tratar los problemas de la desintegración socio-cultural de la sociedad contemporánea, sería impotente para evitarla.

Una vez más los límites previstos para este trabajo no son compatibles ni siquiera con una breve discusión del citado juego de alternativas en prospectiva. Sólo sugeriré que, como una línea de particla, la alternativa "central" parece ser la que ocurra con más probabilidad. Como ya se ha mencionado anteriormente, el conocimiento del riesgo atómico y la precaución al tratar del mismo, así como también la comprensión general incipiente, pero ya clara, del riesgo ecológico, son buenas indicaciones en favor de la probabilidacl de que los grupos gobernantes del mundo tratarán de evitar una catástrofe atómica o ecológica. Suponienclo, ahora, en un segundo

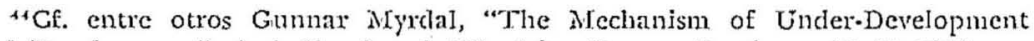
and Development", Apéndice 2, vol. III, Asian Drama, Pantheon, N. Y. 1968, ver también Paul Baran, The Political Economy of Growth, part. cap. V a VII. Monthly Review Press, N. Y., 1959, Maurice Dobb, Capitalism, Developnent and Planning, caps. I y 2, International Publishers, N. Y., 1967.

${ }^{45} \mathrm{Cf}$. François Perroux: L'Economic du XXeme Siècle, partie. erd. Part. Press Univ. de France, 2da. cclic. 1964. Ver también para el caso especifico de América latina, Raul Prebisch, Informe al Inter American Development Bank (Banco Interamericano de Desarrollo), cap. I, Santiago, 1970. 
Helio Jaguaribe / El equilibrio ecológico mundial y los paises subdesariollados

nivel conjetural, que tales políticas atómicas y ecológicas fueran consistentemente implementadas, la gran cuestión restante concierne a los riesgos involucrados en la tercera hipótesis arriba consignada. ¿Están preparados los Centros Imperiales y sus líderes, así como también las clases gobernantes $y^{\prime}$ grupos, en todo el mundo, para tratar la triple falta de uniformidad que plantea la integración socio-cultural de la sociedad contemporánea?

Ia práctica histórica, con respecto a esta cuestión crucial, se hat inclinaclo consistemente en el senticlo de la explotación social e internacional. $\mathrm{Ni}$ las grandes religiones ni los preceptos éticos han sido capaces de contener tal propensión del hombre cuanclo las concliciones se lo han permitido. Es cierto, sin embargo, como ya se indicó en este trabajo, que el desarrollo tecnológico, junto con el carácter totalizador de la sociedad contemporánea, han suprimido la existencia de "proletariados externos explotables", haciendo que la explotación social e internacional, últimamente, sea una práctica que se auto-derrota por los centros y grupos gobernantes. ¿Induciria ese cambio sustancial de concliciones también a un cambio correspondiente en conductas?

Dos aspectos de esa cuestión merecen un comentario muy rápido. El primero concierne al hecho de que en los centros metropolitanos más avanzados la explotación social interna ha cambiado manifiestamente de forma y aun de contenido. Como ya se ha anticipado con clariclad en este trabajo, hay una tendencia evidente, en aquellos centros, a aumentar el nivel actual de consumo de las masas y a reducir sustancialmente la diferencia de consumo entre las clases. Esta tendencia podría llamarse una tendencia hacia la democratización del consumo. En cambio, también resulta manifiesto que en esas mismas socieclades más avanzadas la maquinaria de la clemocracia política es más y más ritualista y menos efectivamente significativa. Formas sutiles (o no tan sutiles) de co-opciones de hecho determinan el modo por el cual se regula el acceso a las más altas funciones con poder de decisión y la renovación de cuerpos realmente importantes con ese mismo poder. Ista tendencia podría llamarse una tendencia hacia la oligarquización de las decisiones, que corre paralela a la otra tendencia ya mencionada ${ }^{40}$. Hay sólidas razones para creer que estas dos tendencias, ahora internamente predominantes en los Estados Unidos y en la Unión Soviética, así como también en el Japón y en los países europeos más avanzados, van a prevalecer a nivel internacional.

$\mathrm{El}$ segundo aspecto que requiere un breve comentario concierne al problema del impacto de consideraciones ecológicas y de escasez

${ }^{40} \mathrm{CF}$. en mi próximo Jibro Political Development, cap. 27, "The Problem of American Democracy". 
de materia prima sobre las relaciones entre los Centros Imperiales y sus periferias y, genéricamente, entre las naciones y grupos desarroIlados y subdesarrollados en el mundo. En ese aspecto, es particularmente de temer que, confrontados con varias formas de escasez ẹcológica, los Centros Imperiales y las naciones y grupos desarrollados reservarán todas o casi todas las facilidades escasas para su propio uso, mientras impondrán al resto del mundo las politicas restrictivas sobre la expansión demográfica, económica y tecnológica requeridas para devolver al mundo el equilibrio ecológico 4 .

Ninguno de los dos aspectos arriba comentados autoriza una visión optimista de la re-integración socio-cultural de la sociedad contemporánea, en condiciones de crisis ecológica. A menos que medidas muy oportunas y eficientes sean capaces de restablecer el equilibrio ecológico y evitar la futura escasez de recursos naturales, los afortunados efectos "anti-explotativos" resultantes del desarrollo de la tecnología tenderán a ser anulados por otro orden de juego "suma-cero", el juego de intentar una supervivencia egoísta en condiciones de dificultades ecológicas. $\mathrm{El}$ hecho de que tal intento es una solución que se derrota a sí misma, en tanto que agrava la desintegración socio-cultural de la sociedad contemporánea, no es por sí mismo capaz de evitar su adopción.

\section{GONGLUSTONES}

Como conclusión a esta breve discusión de las tesis del CoR y los problemas que implican, yo sugeriría en forma clara, imperativa y urgente las tres siguientes necesiclades:

\section{La necesidad de mayor conocimiento}

Se necesita más conocimiento, en términos de datos básicos y más aún en términos de un tratamiento analítico y sistemático de la naturaleza de las variables que intervienen y de su interacción, concerniente a todos los aspectos involucrados en los estudios del CoR, en parte comentados brevemente en este trabajo;

2. La necesidad de formular politicas adecuadns.

Es necesario intentar la formulación y discusión de políticas y estrategias responsables, realistas y humanisticas para que se prevean con tiempo los tres resultados suicidas discutidos en la Parte III de este trabajo (holocausto atómico, ruina eco40-41.

"Cf. James Bonner; ascyeraciones en The Next Ninety Years, op. cit, pp. 
Felio Jaguaribo / Til equilibrio ecologico mundial y los paises subdesarrollados

lógica y desintegración socio-cultural de la sociedad), concibiendo tales políticas en el senticlo de hacer el mejor uso, mientras sea posible, de los nuevos efectos "anti-explotativos" contenidos en la tecnología moderna;

3. La necesidad de acción preventiva

Es indispensable comprometerse en una acción preventiva seria con la iniciativa de gente competente, en forma privada (tal como el Club de Roma) y por medio de la movilización de agencias públicas internacionales adecuadas (tales como varios cuerpos de la UN), con el fin de promover la demanda y la formulación de las políticas, y las condiciones para su implementación eficiente requeridas para los propósitos arriba mencionados. 